\title{
Percentage of nonoverlapping corrected data
}

\author{
Rumen Manolov and Antonio Solanas \\ University of Barcelona, Barcelona, Spain
}

\begin{abstract}
In the present study, we proposed a modification in one of the most frequently applied effect-size procedures in single-case data analysis: the percentage of nonoverlapping data. In contrast with other techniques, the calculus and interpretation of this procedure are straightforward and can be easily complemented by visual inspection of the graphed data. Although the percentage of nonoverlapping data has been found to perform reasonably well in $N=1$ data, the magnitude of effect estimates that it yields can be distorted by trend and autocorrelation. Therefore, the data-correction procedure focuses on removing the baseline trend from data prior to estimating the change produced in the behavior as a result of intervention. A simulation study was carried out in order to compare the original and the modified procedures in several experimental conditions. The results suggest that the new proposal is unaffected by trend and autocorrelation and that it can be used in case of unstable baselines and sequentially related measurements.
\end{abstract}

Single-case designs are useful for obtaining scientific evidence about intervention effectiveness in different behavioral fields of knowledge (Crane, 1985; Gedo, 2000; Tervo, Estrem, Bryson-Brockman, \& Symons, 2003). Recent methodological research on single-case data analysis has centered on effect-size measures instead of on statistical techniques yielding $p$ values exclusively. The increased interest in quantifying the magnitude of effect might be due to the recommendations for reporting studies' results (Wilkinson \& the Task Force on Statistical Inference, 1999) based on the advantages of effect sizes over statistical significance, such as the focus on the strength of relationship between the intervention and behavior of interest, the possibility of establishing different degrees of treatment effectiveness, and the avoidance of sample-size dependence (Cohen, 1990, 1994; Kirk, 1996; Kromrey \& Foster-Johnson, 1996; Rosnow \& Rosenthal, 1989). The importance of effect-size measurements in single-case designs has been reflected in an increased number of recent publications, answering the need for evidence-based interventions in the behavioral sciences (Jenson, Clark, Kircher, \& Kristjansson, 2007; Schlosser \& Sigafoos, 2008; Shadish, Rindskopf, \& Hedges, 2008).

From the perspective of an applied researcher in clinical, educational, or social settings, a potentially useful effect-size index needs to meet several criteria: (1) it must perform well in a short data series, producing low estimates in absence of a treatment effect and higher ones in its presence; (2) it must be easy to interpret in applied rather than in statistical terms; (3) related to the previous criterion, it is desirable that the procedure be designed specifically for $N=1$ data in order to avoid interpretations that are based on group designs terminology; (4) it should be simple to compute, not requiring expertise, commercial statistical software packages, or an excessive amount of time; (5) it should be easily complemented by visual inspection, considering its utility (Parker, Cryer, \& Byrns, 2006) and its frequent application (Kratochwill \& Brody, 1978; Parker \& Brossart, 2003).

As regards the first criterion mentioned, several regression-based techniques have been found to have unacceptable statistical properties (Beretvas \& Chung, 2008; Manolov \& Solanas, 2008; Parker \& Brossart, 2003). These procedures also require a greater amount of knowledge and calculus on the part of the researcher in comparison with the proposed indices related to visual analysis. With respect to the latter procedures, Ma's (2006) percentage of data points exceeding the median and Parker, Hagan-Burke, and Vannest's (2007) percentage of all nonoverlapping data (PAND) were designed to improve the performance of the percentage of nonoverlapping data (PND; Scruggs, Mastropieri, \& Casto, 1987) procedure, but it has been shown that this does not always work (Manolov, Solanas, \& Leiva, in press). Additionally, the magnitude of the effect estimate produced by PAND has a less straightforward interpretation, whereas Pearson's $\Phi^{2}$, which can be obtained from it, requires several steps to be carried out with different software programs (Schneider, Goldstein, \& Parker, 2008).

Given these considerations, the PND procedure that was designed for single-case data can be regarded as a procedure that works well (i.e., better than its most similar alternatives, although not optimally), being simple to interpret and to compute, and closely related to visual inspection. In recent studies, PND has been the most frequently applied procedure for quantifying treatment effectiveness in single-case studies and also in meta-analyses (Schlosser, Lee, \& Wendt, 2008). Nevertheless, despite its attractiveness to psychologists, PND is not trouble-free (Allison \&

R. Manolov, rrumenov13@ub.edu 
Gorman, 1994; Manolov \& Solanas, 2008). Therefore, the main objective of the present investigation was to propose a modification of the PND procedure that was intended to overcome some of its limitations. The performance of the modified index is tested in the context of data sets with different characteristics, such as presence or absence of confounding variables (i.e., trend, serial dependence) and of intervention effects. In order to contrast the percentages obtained against known data attributes, Monte Carlo methods were used to construct the data series.

\section{Overcoming the Drawbacks of PND}

The present study proposed a data-correction procedure to be implemented prior to applying the PND. The main aim of the procedure was to eliminate from the data a possible preexisting trend that was not related to the introduction of the intervention. Since the proposal is basically a modification of PND - adding an initial data-correction step-we refer to the procedure as the "percentage of nonoverlapping corrected data" (PNCD). Before a treatment is introduced (i.e., in an $\mathrm{AB}$ design's initial phase), it can be reasonably assumed that the behavior of the individual $(y)$ or group studied is randomly fluctuating around a certain value; that is, $y_{t}=\varepsilon_{t}$. If there is a trend in the behavior, then $y_{t}=\beta \cdot t+\varepsilon_{t}$, where $\beta$ is the trend coefficient (equal to 0 in the absence of trend), and $t$ is the value of the time variable. The original phase A consists of $n_{\mathrm{A}}$ data points, which, when differenced, leads to a new series of $n_{\mathrm{A}}-1$ values: $\Delta y_{t+1}=$ $y_{t+1}-y_{t}$. In case there is a trend in data,

$$
\begin{aligned}
\Delta y_{t+1} & =\left[\beta \cdot(t+1)+\varepsilon_{t+1}\right]-\left[\beta \cdot t+\varepsilon_{t}\right] \\
& =\beta \cdot t+\beta+\varepsilon_{t+1}-\beta \cdot t-\varepsilon_{i} \\
& =\beta+\varepsilon_{t+1}-\varepsilon_{t} .
\end{aligned}
$$

$\varepsilon_{t+1}$ and $\varepsilon_{t}$ are supposed to be independent and randomly and identically distributed, and their mathematical expectancy is assumed to be 0 . Given that $E\left[\hat{\beta}+\varepsilon_{t+1}-\varepsilon_{t}\right]=\hat{\beta}$, an estimate of $\beta$ can be obtained averaging the differenced data series; that is, $\overline{\Delta y}$ is used as $\hat{\beta}$. After the trend in the baseline phase is estimated, the whole series (both phases $\mathrm{A}$ and $\mathrm{B}$ ) can be corrected by subtracting $\hat{\beta} \cdot t$ (the trend estimate multiplied by the measurement time) from the original data points. This operation is expected to remove any trend from the data and thus to avoid inflation in the percentages obtained by means of PND. Trend is not estimated from the whole data series, since a change in level between the phases may be confounded for trend, and such a correction may remove the intervention effect. The steps necessary for computing both PND and PNCD are illustrated in a following section. Additionally, R codes were developed for computing both indices and are presented in Appendices A and B for interventions aiming to increase and decrease the response rate, respectively.

Concerning autocorrelation, a difference needs to be established between positive serial dependence and negative serial dependence. Higher degrees of positive autocorrelation can be represented by upward or downward trends and, therefore, one can conjecture that a correction focusing on trend may also have influence on it and attenuate its impact on the effect-size index. Negative autocorrelation, however, is related to alternations of dissimilar measurements. In this case, the effect of the correction procedure proposed cannot be foreseen and needs to be explored.

Outliers represent another data feature that can distort the magnitude of effect estimates provided by PND. For instance, a single extremely high value in phase A can mask a behavioral change taking place after the treatment is introduced. Outliers can be detected using statistical calculi and can be controlled by means of elimination, winsorization, and so on. However, it has to be taken into account that in a single-case study, the applied researcher possesses a thorough knowledge of the client and is able to identify which measurement is an extreme and potentially anomalous one and interpret it (e.g., seek for its reason) from a clinical, educational, social, and so on, point of view. Such a theoretical interpretation may be more meaningful than an arbitrary statistical treatment of the unexpected datum.

\section{METHOD}

\section{AB Series' Lengths}

Short data series $\left(N=n_{\mathrm{A}}+n_{\mathrm{B}}\right)$ were included in the present study, since those are more feasible in applied settings: (1) $N=10$, with $n_{\mathrm{A}}=n_{\mathrm{B}}=5$; (2) $N=15$, with $n_{\mathrm{A}}=5 ; n_{\mathrm{B}}=10$; (3) $N=15$, with $n_{\mathrm{A}}=7 ; n_{\mathrm{B}}=8 ;$ (4) $N=20$, with $n_{\mathrm{A}}=n_{\mathrm{B}}=10 ;(5) N=30$, with $n_{\mathrm{A}}=n_{\mathrm{B}}=15$; and (6) $N=40$, with $n_{\mathrm{A}}=n_{\mathrm{B}}=20$.

\section{Data Generation}

For each combination of $n_{\mathrm{A}}$ and $n_{\mathrm{B}}$, the data were according to the model proposed by Huitema and McKean (2000, 2007b): $y_{t}=$ $\beta_{0}+\beta_{1} \cdot T_{t}+\beta_{2} \cdot D_{t}+\beta_{3} \cdot S C_{t}+\varepsilon_{t}$, where $y_{t}$ is the value of the dependent variable at moment $t ; \beta_{0}$ is the intercept set to $0 ; \beta_{1}, \beta_{2}$, and $\beta_{3}$ are the coefficients associated with trend, level change, and slope change, respectively; $T_{t}$ is the value of the time variable at moment $t$ (taking values from 1 to $N$ ); $D_{t}$ is a dummy variable for level change (equal to 0 for phase $\mathrm{A}$ and to 1 for phase $\mathrm{B}$ ); $S C_{t}$ is the value of the slope change variable being equal to 0 for phase $A$, and taking values from 0 to $\left(n_{\mathrm{B}}-1\right)$ for phase $\mathrm{B}$; and $\varepsilon_{t}$ is the error term.

The error term $\left(\varepsilon_{t}\right)$ was generated following two different models. One of these was the commonly used first-order autoregressive model $\varepsilon_{t}=\phi_{1} \cdot \varepsilon_{t-1}+u_{t}$, with $\phi_{1}$ ranging from -.9 to .9 in steps of . 1 . Since there is evidence that other models - especially a first-order moving average - can be used to represent behavioral data (Harrop \& Velicer, 1985), the MA(1) model, $\varepsilon_{t}=u_{t}-\theta_{1} \cdot u_{t-1}$, presented in McCleary and Hay (1980) was studied using 19 values of $\theta_{1}$ : - .9(.1).9. According to the formula $\phi_{1}=-\theta_{1} /\left(1+\theta_{1}^{2}\right)$, this meant that the degrees of autocorrelation ranged from -.4972 to .4972 .

For both models, the random variable $u_{t}$ was generated following $N(0,1)$ and an exponential and a uniform distribution with the same mean and standard deviation, since normal distributions are not always appropriate models for behavioral measurements (Bradley, 1977; Micceri, 1989). The aforementioned distributions are relevant, since they differ in terms of skewness and kurtosis from the Gaussian distribution.

The values of $\beta_{1}, \beta_{2}$, and $\beta_{3}(.06, .3$, and .15 , respectively) were chosen by trial and error - a procedure that was also followed by Parker and Brossart (2003) and Brossart, Parker, Olson, and Mahadevan (2006), aiming to avoid floor and ceiling effects in the percentages obtained (Manolov \& Solanas, 2008). In addition, the values of those coefficients were determined in a way to produce equivalent mean shifts in the case of trend, a change in slope, and a change in level for the $n_{\mathrm{A}}=n_{\mathrm{B}}=5$ data series. In any case, the specific beta values are not essential, since they only serve to construct data series with and without trend or intervention effect and thus create a common background for comparing PND and PNCD. 


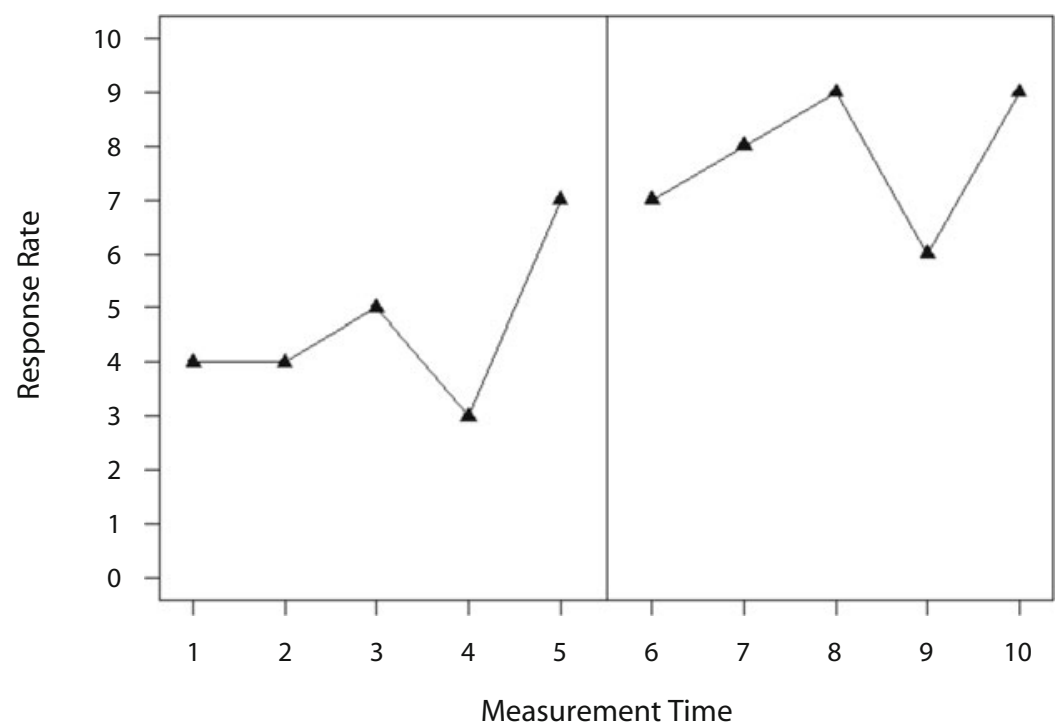

Figure 1. A fictitious example of an $\mathrm{AB}$ data series with $n_{\mathrm{A}}=n_{\mathrm{B}}=5$.

\begin{abstract}
Analysis
Prior to presenting in detail the steps needed to carry out the two effect-size procedures included in the present study, an example of a fictitious data set is presented. Consider a psychological single-case study educating a parent to interact with children who have been diagnosed with autism, counting a child's desirable behavior of interest (e.g., communication) in each session (Symon, 2005). The data gathered using the $\mathrm{AB}$ design structure $(4,4,5,3$, and 7 positive communications during baseline and $7,8,9,7$, and 9 during treatment phase) can be represented graphically, as is shown in Figure 1. In the following section, the original and the proposed procedures are applied to the data set presented in order to illustrate their calculus.
\end{abstract}

\section{PND}

(1) Identify the highest measurement in phase A. In the example, it is 7 positive communications corresponding to baseline day 5 .

(2) Calculate the number of phase B data points that exceed the value identified in the previous step. The measurements corresponding to days 7,8 , and 10 are greater than 7 , so there are 3 values exceeding phase A's highest value.

(3) Divide the value obtained in step 2 by the number of observations in phase $\mathrm{B}$. The number of phase $\mathrm{B}$ observations is 5 , and the result of the division is $3 / 5=.6$.

(4) Multiply the value obtained in step 3 by 100 in order to convert the proportion into a percentage. The percentage obtained for the example is $.6 \cdot 100=60 \%$.

\section{PNCD}

(1) Difference the phase A data points and obtain the differenced series with the length $n_{\mathrm{A}}-1$. In the example, the differenced series has the following $5-1=4$ data points: $0(4-4)$, $1(5-4),-2(3-5)$, and $4(7-3)$.

(2) Compute the mean of the differenced series. The average of $0,1,-2$, and 4 is 0.75 .

(3) Compute the trend-correction factor for each data point: the mean of the differenced series, multiplied by $T_{t}$. In the example, the values of the correction factor are $.75 \cdot 1, .75 \cdot 2, \ldots, .75 \cdot 10$.

(4) Perform the data correction subtracting the corresponding correction factor from each original data point. After the correction phase, $A$ consists of $3.25(4-.75 \cdot 1) ; 2.5(4-.75 \cdot 2) ; 2.75$ $(5-.75 \cdot 3) ; 0(3-.75 \cdot 4)$; and $3.25(7-.75 \cdot 5)$. Phase B consists of the following data points: $2.5(7-.75 \cdot 6) ; 2.75(8-.75 \cdot 7)$; $3(9-.75 \cdot 8) ; .25(7-.75 \cdot 9)$; and $1.5(9-.75 \cdot 10)$.

(5) Apply PND: None of the phase B data points is greater than the phase A highest value (3.25) and, therefore, $\mathrm{PNCD}=0 \%$.

\section{Simulation}

The specific steps that were implemented in the Fortran programs (one for each of the six series' length) were the following:

(1) Systematic selection of each of the 19 values of $\phi_{1}$ or $\theta_{1}$.

(2) Systematic selection of the $\left(\beta_{1}, \beta_{2}\right.$, and $\left.\beta_{3}\right)$ parameters for data generation, leading to eight different data patterns: autoregressive or moving average model with no effect or trend, trend, level change, slope change, trend and level change, trend and slope change, combined level and slope change, and trend and combined level and slope change.

(3) 100,000 iterations of steps 4-15.

(4) Generate the $u_{t}$ term according to an exponential, normal, or uniform distribution, eliminating the first 50 random numbers using the next $N$ ones.

(5) Establish $\varepsilon_{1}=u_{1}$.

(6) Obtain the error term $\varepsilon_{t}$ out of the random variable $u_{t}$ using the $\operatorname{AR}(1)$ model $\varepsilon_{t}=\phi_{1} \cdot \varepsilon_{t-1}+u_{t}$ or the MA(1) model $\varepsilon_{t}=$ $u_{t}-\theta_{1} \cdot u_{t-1}$.

(7) Obtain the time array $T_{t}=1,2, \ldots, N$.

(8) Obtain the dummy treatment variable array $D_{t}$, where $D_{t}=0$ for phase $\mathrm{A}$ and $D_{t}=1$ for phase $\mathrm{B}$.

(9) Obtain the slope change array according to $S C_{t}=\left[T_{t}-\right.$ $\left.\left(n_{\mathrm{A}}+1\right)\right] \cdot D_{t}$.

(10) Obtain the $y_{t}$ array containing measurements (i.e., dependent variable): $y_{t}=\beta_{0}+\beta_{1} \cdot T_{t}+\beta_{2} \cdot D_{t}+\beta_{3} \cdot S C_{t}+\varepsilon_{t}$.

(11) Calculate PND on the original data (i.e., the $y_{t}$ array).

(12) Correct the data according to the procedure proposed.

(13) Calculate PNCD on corrected data.

(14) Average the obtained percentages from the 100,000 replications of each experimental condition.

For data generation NAG libraries, nag_rand_neg_exp, nag_ rand_normal, and nag_rand_uniform were used. In order to guarantee suitable simulated data, the 50 values previous to each simulated data series were eliminated in order to reduce artificial effects (Greenwood \& Matyas, 1990) and to avoid dependence between successive data series (Huitema, McKean, \& McKnight, 1999). 


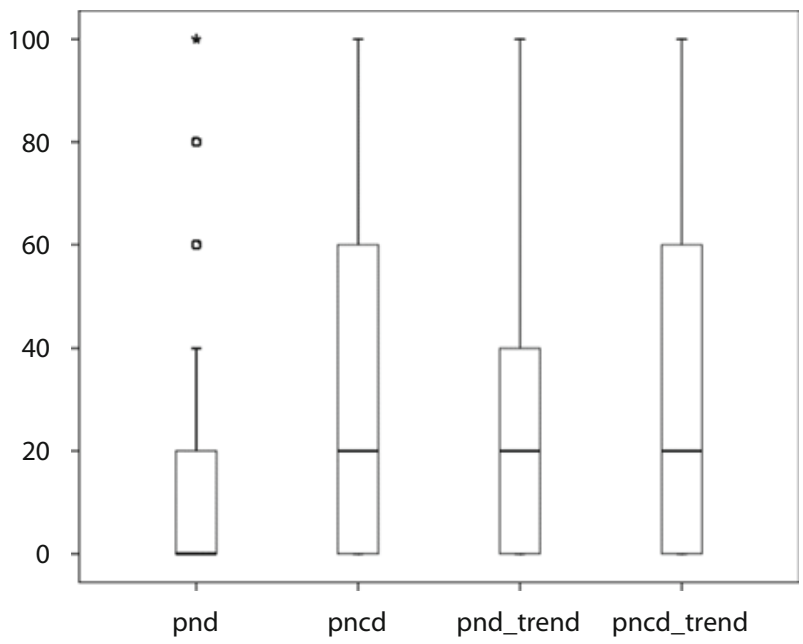

Figure 2. Distribution of the percentages provided by the percentage of nonoverlapping data (PND) and the percentage of nonoverlapping corrected data (PNCD) in the absence (the two boxplots on the left) and presence (the two boxplots on the right) of trend. The percentages were taken from 100,000 samples of independent $n_{\mathrm{A}}=n_{\mathrm{B}}=\mathbf{5}$ data, with no treatment effect simulated and normal error.

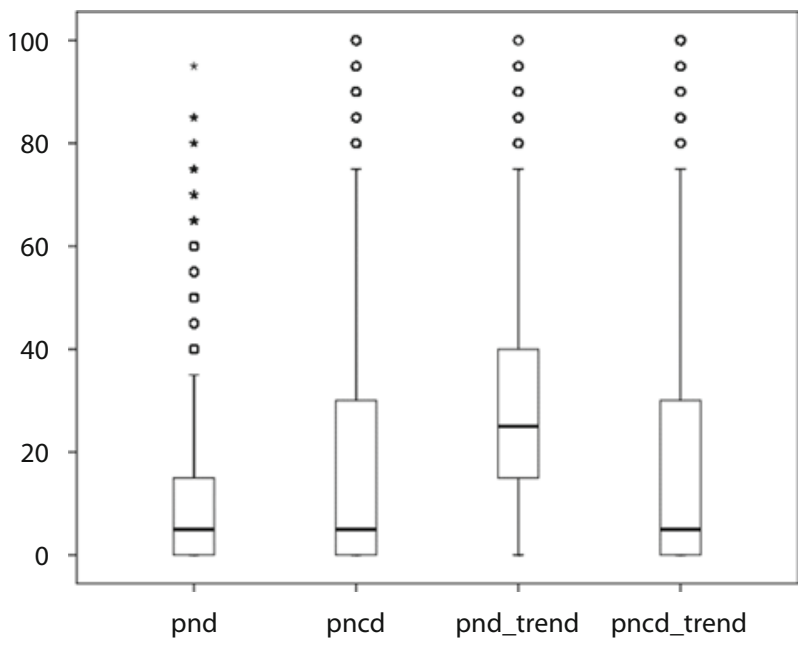

Figure 3. Distribution of the percentages provided by the percentage of nonoverlapping data (PND) and the percentage of nonoverlapping corrected data (PNCD) in the absence (the two boxplots on the left) and presence (the two boxplots on the right) of trend. The percentages were taken from 100,000 samples of $n_{\mathrm{A}}=n_{\mathrm{B}}=20$ data, with level change simulated and uniform error in moving average processes with autocorrelation of .5 .

\section{RESULTS}

When the data series represent solely random fluctuation (i.e., there is no trend, autocorrelation, or treatment effect), the percentages provided by PNCD are systematically larger than the ones provided by PND, as is illustrated by Figure 2. This finding implies that PND may be a better filter for ineffective interventions in the absence of trend and serial dependence. In the aforementioned conditions, higher effect-size estimates were also obtained for PNCD than for PND when treatment effects existed. However, if the data represent a trend, the PND estimates increase and may become superior to the PNCD estimates for both independent (Figure 2 ) and serially related (Figure 3 ) data series, as the within-figure comparisons show.

\section{Trend Effect}

In order to quantify the distortion of effect-size estimates produced by trend, the ratio between percentages with and without trend in data was computed. Therefore, a ratio close to 1 would indicate that trend does not introduce distortion, whereas values greater than 1 would imply an overestimation of the magnitude of effect. In the experimental conditions with no treatment effect simulated (Table 1), ratios $>1$ entail an increment in false alarms, which is the case for PND in contrast with PNCD, which maintains approximately the same magnitude estimates in both the presence and absence of trend. This finding is applicable to all series lengths and errors' distributions tested.

When there is a treatment effect (slope change, level change, or both), the presence of trend leads to the overestimation of the effect size obtained through PND, as Table 2 shows. In contrast, the estimates provided by PNCD are not affected by the confounding variable.

The ratios presented in Tables 1 and 2 show that the PND estimates become more distorted by trend when the number of measurements $N$ increases. PNCD seems to deal effectively with trend for both shorter and longer data series.

\section{Autocorrelation Effect}

The distortion of effect-size estimates produced by serial dependence was quantified by means of the ratio

Table 1

Distortion As a Result of Trend in an Independent Data Series

\begin{tabular}{|c|c|c|c|}
\hline \multicolumn{2}{|c|}{ Phase Length } & \multicolumn{2}{|c|}{$\begin{array}{c}\text { Ratio Trend/ } \\
\text { Random Fluctuations }\end{array}$} \\
\hline$n_{\mathrm{A}}$ & $\overline{n_{\mathrm{B}}}$ & PND & PNCD \\
\hline \multicolumn{4}{|c|}{ Exponential } \\
\hline 5 & 5 & 1.336 & 0.996 \\
\hline 5 & 10 & 1.576 & 1.002 \\
\hline 7 & 8 & 1.570 & 1.003 \\
\hline 10 & 10 & 1.807 & 0.999 \\
\hline 15 & 15 & 2.431 & 1.000 \\
\hline 20 & 20 & 3.293 & 0.995 \\
\hline \multicolumn{4}{|c|}{ Normal } \\
\hline 5 & 5 & 1.429 & 0.998 \\
\hline 5 & 10 & 1.674 & 1.000 \\
\hline 7 & 8 & 1.772 & 0.997 \\
\hline 10 & 10 & 2.279 & 1.000 \\
\hline 15 & 15 & 3.601 & 1.003 \\
\hline 20 & 20 & 5.511 & 1.005 \\
\hline \multicolumn{4}{|c|}{ Uniform } \\
\hline 5 & 5 & 1.517 & 1.002 \\
\hline 5 & 10 & 1.747 & 0.997 \\
\hline 7 & 8 & 2.003 & 0.995 \\
\hline 10 & 10 & 2.761 & 1.005 \\
\hline 15 & 15 & 4.590 & 0.991 \\
\hline 20 & 20 & 6.952 & 0.993 \\
\hline
\end{tabular}

Note-The values represent the ratio between the presence of trend/ absence of trend in experimental conditions without treatment effect. $\mathrm{PND}$, percentage of nonoverlapping data; $\mathrm{PNCD}$, percentage of nonoverlapping corrected data. 
Table 2

Distortion As a Result of Trend in Independent Data Series

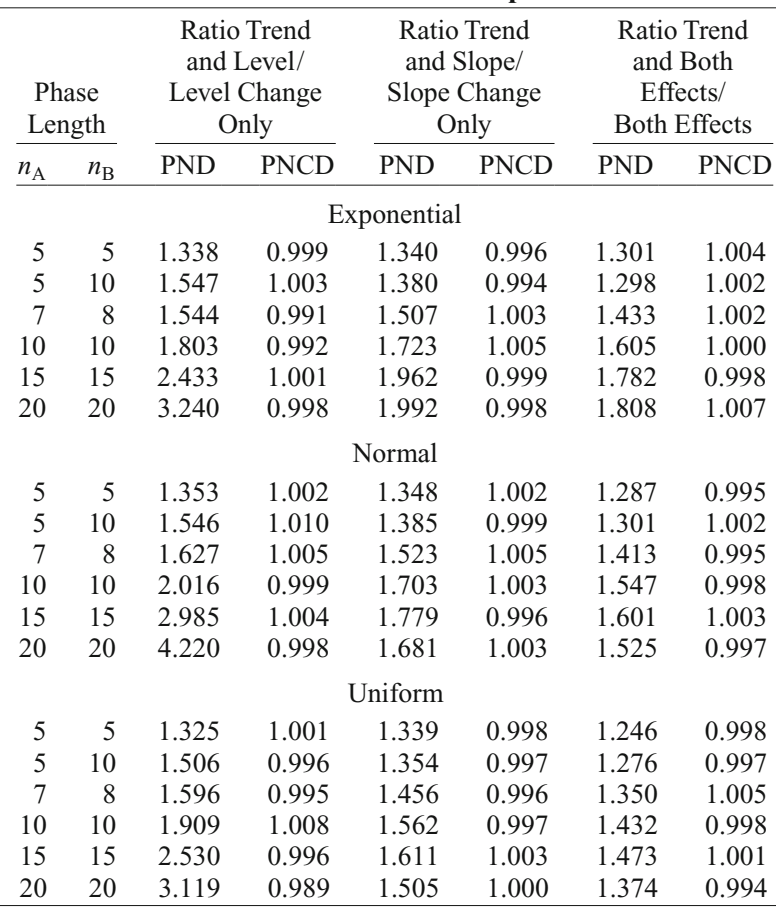

Note-The values represent the ratio between the presence of trend/ absence of trend in experimental conditions with single or combined treatment effect. PND, percentage of overlapping data; $\mathrm{PNCD}$, percentage of nonoverlapping corrected data.

between percentages computed for autocorrelated and independent data. Once again, ratios of 1 imply no distortion, and values greater than 1 are indicative of elevated false alarm rates in the absence of an intervention effect. In the case of exponential errors, for both $\mathrm{AR}(1)$ and MA(1) models, PNCD performs worse than PND when there is negative autocorrelation, and performs only slightly better for positive serial dependence. In contrast, for the normal and uniform errors, PNCD outperforms PND. For these two error distributions and AR(1) processes (Table 3) with $\phi_{1}>0$, the difference between PNCD and PND increases for longer data series, whereas for $\phi_{1}<0$ PNCD performs better only for $N \leq 20$. For the MA(1) processes (Table 4) with negative values of $\theta_{1}$ (i.e., positive autocorrelation), PNCD shows less distortion than PND, whereas for $\theta_{1}>0$, it outperforms PND only for $N \leq 15$, always referring to normal and uniform errors.

\section{Combined Effect}

In addition to the individual effects of each of these data features, their combined effect was studied following the same procedure for quantifying distortion. Table 5 shows that for AR(1) processes with trend, PNCD is much less affected by the confounding variables than is PND, whose effect size estimate is quintupled in certain experimental conditions. For MA(1) processes (Table 6), the findings are similarly favorable for PNCD.
Table 3

Distortion As a Result of an AR(1) Process

\begin{tabular}{|c|c|c|c|c|c|c|c|}
\hline \multicolumn{2}{|c|}{$\begin{array}{l}\text { Phase } \\
\text { Length }\end{array}$} & \multicolumn{2}{|c|}{$\begin{array}{c}\text { Ratio } \\
\phi_{1}=-.3 / \\
\text { Random } \\
\text { Fluctuations }\end{array}$} & \multicolumn{2}{|c|}{$\begin{array}{c}\text { Ratio } \\
\phi_{1}=.3 / \\
\text { Random } \\
\text { Fluctuations }\end{array}$} & \multicolumn{2}{|c|}{$\begin{array}{c}\text { Ratio } \\
\phi_{1}=.6 / \\
\text { Random } \\
\text { Fluctuations }\end{array}$} \\
\hline$n_{\mathrm{A}}$ & $n_{\mathrm{B}}$ & PND & PNCD & PND & PNCD & PND & PNCD \\
\hline \multicolumn{8}{|c|}{ Exponential } \\
\hline 5 & 5 & 0.941 & 0.926 & 1.135 & 1.121 & 1.302 & 1.250 \\
\hline 5 & 10 & 0.948 & 0.943 & 1.169 & 1.110 & 1.422 & 1.234 \\
\hline 7 & 8 & 0.955 & 0.943 & 1.167 & 1.147 & 1.482 & 1.365 \\
\hline 10 & 10 & 0.958 & 0.940 & 1.164 & 1.157 & 1.559 & 1.455 \\
\hline 15 & 15 & 0.956 & 0.950 & 1.138 & 1.157 & 1.591 & 1.511 \\
\hline 20 & 20 & 0.981 & 0.953 & 1.141 & 1.152 & 1.614 & 1.545 \\
\hline \multicolumn{8}{|c|}{ Normal } \\
\hline 5 & 5 & 0.933 & 0.953 & 1.158 & 1.065 & 1.379 & 1.121 \\
\hline 5 & 10 & 0.933 & 0.968 & 1.167 & 1.042 & 1.441 & 1.093 \\
\hline 7 & 8 & 0.946 & 0.955 & 1.171 & 1.067 & 1.503 & 1.173 \\
\hline 10 & 10 & 0.944 & 0.953 & 1.174 & 1.069 & 1.579 & 1.207 \\
\hline 15 & 15 & 0.965 & 0.954 & 1.178 & 1.075 & 1.637 & 1.221 \\
\hline 20 & 20 & 0.983 & 0.957 & 1.168 & 1.065 & 1.634 & 1.212 \\
\hline \multicolumn{8}{|c|}{ Uniform } \\
\hline 5 & 5 & 0.929 & 0.961 & 1.158 & 1.060 & 1.378 & 1.114 \\
\hline 5 & 10 & 0.929 & 0.967 & 1.166 & 1.032 & 1.428 & 1.079 \\
\hline 7 & 8 & 0.936 & 0.954 & 1.185 & 1.050 & 1.497 & 1.130 \\
\hline 10 & 10 & 0.932 & 0.946 & 1.195 & 1.050 & 1.561 & 1.146 \\
\hline 15 & 15 & 0.949 & 0.920 & 1.189 & 1.006 & 1.602 & 1.088 \\
\hline 20 & 20 & 0.971 & 0.912 & 1.188 & 0.994 & 1.619 & 1.035 \\
\hline
\end{tabular}

Note-The values represent the ratio between serially dependent data and an independent series with no trend or intervention effect. PND, percentage of nonoverlapping data; PNCD, percentage of nonoverlapping corrected data.
Table 4

Distortion As a Result of an MA(1) Process

\begin{tabular}{|c|c|c|c|c|c|}
\hline \multicolumn{2}{|c|}{$\begin{array}{l}\text { Phase } \\
\text { Length }\end{array}$} & \multicolumn{2}{|c|}{$\begin{array}{c}\text { Ratio } \\
\theta_{1}=-.5 / \\
\text { Random } \\
\text { Fluctuations }\end{array}$} & \multicolumn{2}{|c|}{$\begin{array}{c}\text { Ratio } \\
\theta_{1}=.5 / \\
\text { Random } \\
\text { Fluctuations }\end{array}$} \\
\hline$n_{\mathrm{A}}$ & $n_{\mathrm{B}}$ & PND & PNCD & PND & PNCD \\
\hline \multicolumn{6}{|c|}{ Exponential } \\
\hline 5 & 5 & 0.913 & 0.887 & 1.232 & 1.177 \\
\hline 5 & 10 & 0.903 & 0.914 & 1.242 & 1.151 \\
\hline 7 & 8 & 0.912 & 0.886 & 1.248 & 1.190 \\
\hline 10 & 10 & 0.915 & 0.882 & 1.257 & 1.196 \\
\hline 15 & 15 & 0.925 & 0.910 & 1.240 & 1.206 \\
\hline 20 & 20 & 0.947 & 0.920 & 1.235 & 1.196 \\
\hline \multicolumn{6}{|c|}{ Normal } \\
\hline 5 & 5 & 1.203 & 1.077 & 0.887 & 0.927 \\
\hline 5 & 10 & 1.217 & 1.063 & 0.880 & 0.947 \\
\hline 7 & 8 & 1.226 & 1.080 & 0.901 & 0.919 \\
\hline 10 & 10 & 1.221 & 1.077 & 0.910 & 0.902 \\
\hline 15 & 15 & 1.207 & 1.067 & 0.931 & 0.905 \\
\hline 20 & 20 & 1.197 & 1.058 & 0.945 & 0.899 \\
\hline \multicolumn{6}{|c|}{ Uniform } \\
\hline 5 & 5 & 1.194 & 1.066 & 0.882 & 0.938 \\
\hline 5 & 10 & 1.200 & 1.046 & 0.868 & 0.951 \\
\hline 7 & 8 & 1.207 & 1.047 & 0.881 & 0.925 \\
\hline 10 & 10 & 1.205 & 1.038 & 0.906 & 0.894 \\
\hline 15 & 15 & 1.218 & 0.990 & 0.929 & 0.860 \\
\hline 20 & 20 & 1.179 & 0.947 & 0.940 & 0.842 \\
\hline
\end{tabular}

Note-The values represent the ratio between nonnull and null $\theta_{1}$ parameters in series with no trend or intervention effect. PND, percentage of nonoverlapping data; PNCD, percentage of nonoverlapping corrected data. 
Table 5

Distortion As a Result of Combined Presence of Trend and an AR(1) Process

\begin{tabular}{|c|c|c|c|c|c|c|c|}
\hline & \multicolumn{2}{|c|}{$\begin{array}{c}\text { Ratio Trend and } \\
\phi_{1}=-.3 / \\
\text { Random } \\
\text { Fluctuations }\end{array}$} & \multicolumn{2}{|c|}{$\begin{array}{c}\text { Ratio Trend and } \\
\phi_{1}=.3 / \\
\text { Random } \\
\text { Fluctuations } \\
\end{array}$} & \multicolumn{2}{|c|}{$\begin{array}{c}\text { Ratio Trend and } \\
\qquad \phi_{1}=.6 / \\
\text { Random } \\
\text { Fluctuations } \\
\end{array}$} \\
\hline$n_{\mathrm{A}}$ & $n_{\mathrm{B}}$ & PND & PNCD & PND & PNCD & PND & PNCD \\
\hline \multicolumn{8}{|c|}{ Exponential } \\
\hline 5 & 5 & 1.267 & 0.929 & 1.518 & 1.131 & 1.671 & 1.239 \\
\hline 5 & 10 & 1.484 & 0.942 & 1.785 & 1.116 & 2.007 & 1.240 \\
\hline 7 & 8 & 1.489 & 0.941 & 1.793 & 1.140 & 2.012 & 1.284 \\
\hline 10 & 10 & 1.734 & 0.939 & 2.074 & 1.164 & 2.566 & 1.459 \\
\hline 15 & 15 & 2.336 & 0.951 & 2.734 & 1.150 & 3.368 & 1.524 \\
\hline 20 & 20 & 3.211 & 0.960 & 3.679 & 1.141 & 4.369 & 1.520 \\
\hline \multicolumn{8}{|c|}{ Normal } \\
\hline 5 & 5 & 1.325 & 0.953 & 1.581 & 1.056 & 1.783 & 1.126 \\
\hline 5 & 10 & 1.565 & 0.969 & 1.839 & 1.036 & 2.043 & 1.089 \\
\hline 7 & 8 & 1.663 & 0.950 & 1.961 & 1.068 & 2.223 & 1.170 \\
\hline 10 & 10 & 2.130 & 0.944 & 2.457 & 1.068 & 2.763 & 1.210 \\
\hline 15 & 15 & 3.359 & 0.944 & 3.768 & 1.068 & 3.961 & 1.200 \\
\hline 20 & 20 & 5.100 & 0.955 & 5.594 & 1.075 & 5.585 & 1.215 \\
\hline \multicolumn{8}{|c|}{ Uniform } \\
\hline 5 & 5 & 1.370 & 0.959 & 1.600 & 1.054 & 1.765 & 1.115 \\
\hline 5 & 10 & 1.587 & 0.968 & 1.826 & 1.032 & 1.980 & 1.076 \\
\hline 7 & 8 & 1.795 & 0.952 & 2.056 & 1.049 & 2.205 & 1.133 \\
\hline 10 & 10 & 2.415 & 0.945 & 2.711 & 1.054 & 2.758 & 1.139 \\
\hline 15 & 15 & 4.027 & 0.917 & 4.360 & 1.015 & 4.033 & 1.092 \\
\hline 20 & 20 & 6.132 & 0.904 & 6.590 & 0.995 & 5.766 & 1.037 \\
\hline
\end{tabular}

Note-The values represent the ratio between serially dependent data with trend and independent series with no trend. PND, percentage of nonoverlapping data; PNCD, percentage of nonoverlapping corrected data.

\section{Discrimination Between Data Patterns}

In general, the desirable characteristics of an effect-size procedure are to be sensitive to intervention effects and are not to be affected, for instance, by trend or serial dependence. Hence, an optimal performance (illustrated by Figure 4) would imply (1) low effect-size estimates in the absence of a treatment effect; (2) low effect-size estimates when there is only a general trend; and (3) higher estimates when there are actual changes in the response rate because of an intervention.

Comparing this ideal discrimination with the estimates obtained by means of PND and PNCD, it can be seen that there is a greater resemblance in the case of the latter pro-
Table 6

Distortion As a Result of Combined Presence of Trend and an MA(1) Process

\begin{tabular}{|c|c|c|c|c|c|}
\hline $\begin{array}{l}\text { Phase } \\
\text { Length }\end{array}$ & & \multicolumn{2}{|c|}{$\begin{array}{c}\text { Ratio Trend and } \\
\theta_{1}=-.5 / \\
\text { Random } \\
\text { Fluctuations }\end{array}$} & \multicolumn{2}{|c|}{$\begin{array}{c}\text { Ratio Trend and } \\
\theta_{1}=.5 / \\
\text { Random } \\
\text { Fluctuations }\end{array}$} \\
\hline$n_{\mathrm{A}}$ & $n_{\mathrm{B}}$ & PND & PNCD & PND & PNCD \\
\hline \multicolumn{6}{|c|}{ Exponential } \\
\hline 5 & 5 & 1.217 & 0.888 & 1.597 & 1.174 \\
\hline 5 & 10 & 1.406 & 0.910 & 1.837 & 1.153 \\
\hline 7 & 8 & 1.418 & 0.892 & 1.874 & 1.196 \\
\hline 10 & 10 & 1.658 & 0.877 & 2.153 & 1.184 \\
\hline 15 & 15 & 2.268 & 0.910 & 2.839 & 1.201 \\
\hline 20 & 20 & 3.099 & 0.920 & 3.727 & 1.182 \\
\hline \multicolumn{6}{|c|}{ Normal } \\
\hline 5 & 5 & 1.608 & 1.075 & 1.253 & .924 \\
\hline 5 & 10 & 1.850 & 1.062 & 1.462 & .947 \\
\hline 7 & 8 & 1.962 & 1.078 & 1.562 & .919 \\
\hline 10 & 10 & 2.412 & 1.072 & 1.970 & .909 \\
\hline 15 & 15 & 3.619 & 1.066 & 3.074 & .900 \\
\hline 20 & 20 & 5.231 & 1.052 & 4.601 & .895 \\
\hline \multicolumn{6}{|c|}{ Uniform } \\
\hline 5 & 5 & 1.602 & 1.082 & 1.271 & .933 \\
\hline 5 & 10 & 1.831 & 1.054 & 1.485 & .949 \\
\hline 7 & 8 & 1.974 & 1.056 & 1.617 & .924 \\
\hline 10 & 10 & 2.539 & 1.039 & 2.150 & .891 \\
\hline 15 & 15 & 3.938 & 0.992 & 3.503 & .857 \\
\hline 20 & 20 & 5.815 & 0.949 & 5.281 & .836 \\
\hline
\end{tabular}

Note-The values represent the ratio between moving average data with trend and data series with $\theta_{1}=0$ and no trend. PND, percentage of nonoverlapping data; PNCD, percentage of nonoverlapping corrected data.

cedure. That is, a combined effect (change both in level and in slope) yields a greater effect-size estimate than does an individual effect, and the percentage obtained in the absence of an intervention effect is even lower. Additionally, trend does not shift estimates up, as is the case for PND, which detects trend as an intervention effect. Figure 5 illustrates these findings for the shortest series length studied.

\section{DISCUSSION}

The present investigation proposed a data correction step to be introduced prior to applying the PND as a tech-

Data Series Characteristics: Length, Error Term, etc.

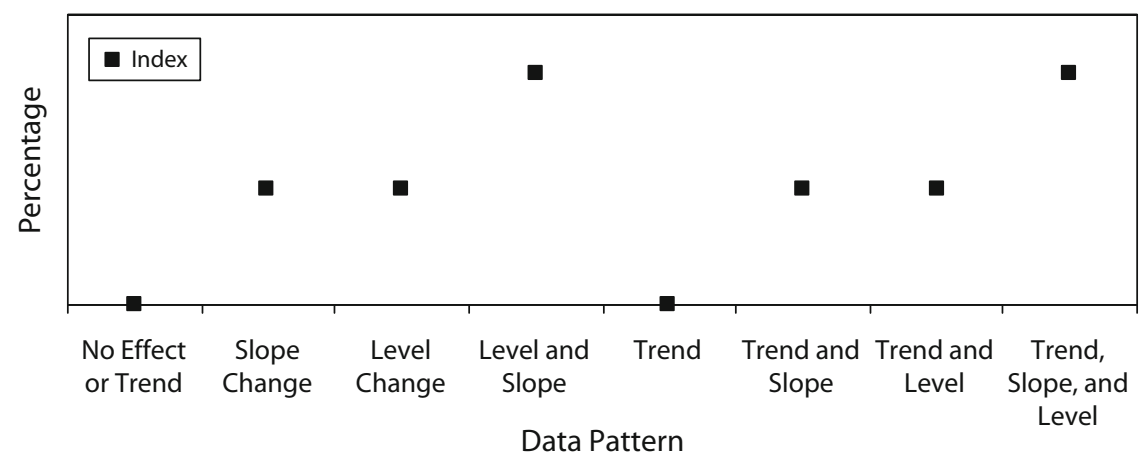

Figure 4. Ideal discrimination between data patterns. 
Independent $5+5$ Series With Normal Error and $\phi=.3$

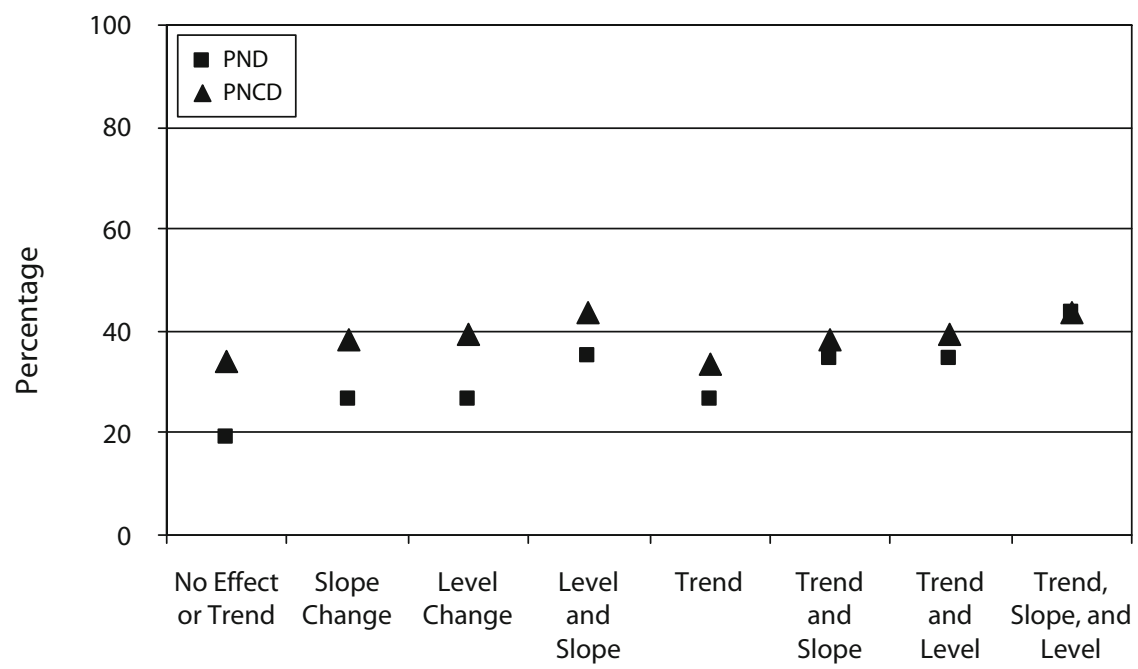

Data Pattern

Independent $5+5$ Series With Uniform Error and $\theta=-.5$

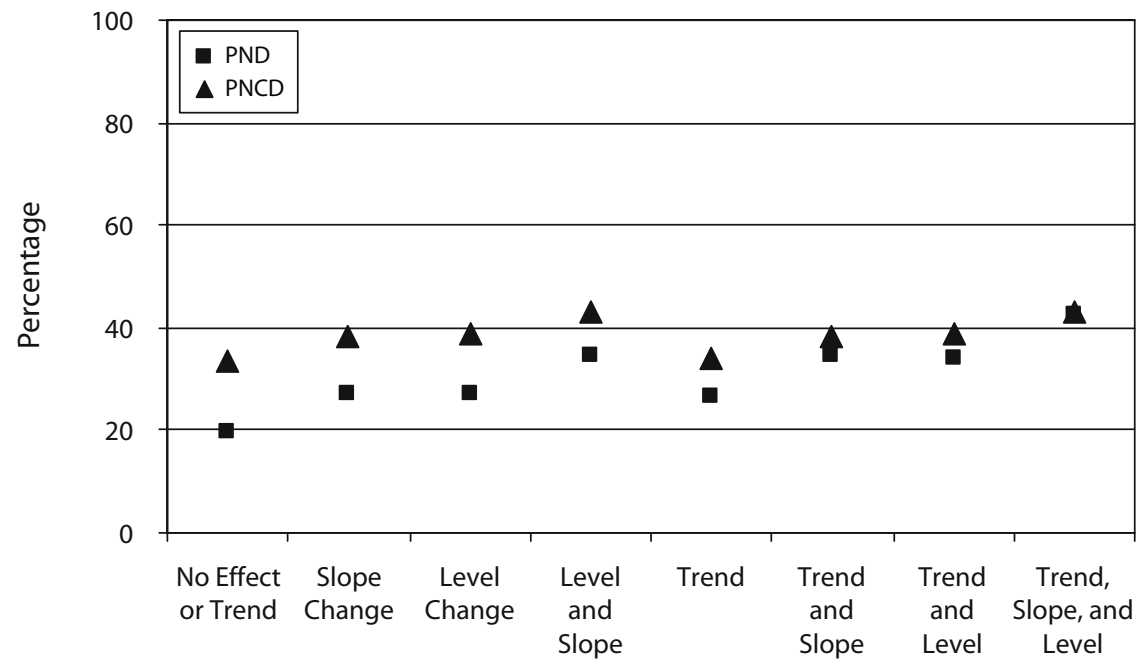

Data Pattern

Figure 5. Discrimination between data patterns for both indices in different experimental conditions. Upper panel: $N=10$ series generated from an AR process with normal error and $\phi_{1}=.3$. Lower panel: $N=10$ series generated from an MA process with uniform error and $\theta_{1}=-.5$.

nique for quantifying treatment effectiveness. The modified procedure was compared with the original in the context of data sets generated with known attributes, such as trend, autocorrelation, and treatment effect. For applied researchers, the results that were obtained suggest that PNCD is an effective method to deal with trend, and that it can, therefore, be used in situations when preintervention measurements are not pure random fluctuation. Unstable baselines have been regarded as undesirable, but they can be common in applied settings in which the introduction of the treatment is subjected to factors that cannot always be controlled by the practitioners. Although a professional might be reluctant to initiate the intervention when there is trend in data, treatment administration may be imposed by institutional time schedules, a client's availability, and so on. In such a case, some kind of statistical control is advisable (Kazdin, 1978), and it can be achieved by means of the procedure proposed in the present article. Apart from behavioral data with baseline trends, another potential context for application of PNCD consists of studies in which the data points are not sufficiently spaced in time and can present a sequential relation. PNCD ought to be preferred to PND in these cases, because of the fact that autocorrelation is more problematic for the latter. 
Whenever the behavioral measurements are not serially dependent and do not present a trend, PND may be a better option than PNCD, since it produces a lower magnitude of effect estimates. This difference in the estimates implies that in the aforementioned cases, PND is less likely to label an intervention as effective when it is not. It has already been discussed that different effect-size procedures may lead to different conclusions about the degree of treatment effectiveness for the same data set (McGrath \& Meyer, 2006; Parker et al., 2005). In the particular case of PND and PNCD, the difference in estimates implies that the interpretation benchmarks proposed by Scruggs and Mastropieri (1998) cannot be applied directly to PNCD. On the other hand, there is evidence that PND is conservative, as compared with other procedures for estimating magnitude of effect (Jenson et al., 2007). Therefore, the effect-size estimates provided by PNCD may resemble more the ones obtained by other models.

From a methodological perspective, PNCD can be regarded as an attempt to improve a procedure that is attractive to applied psychologists and is frequently employed by them. The aim is not only to achieve a better performance, but also to maintain the simplicity of the technique. Therefore, we consider that the modifications balancing statistical properties improvements and low levels of calculus/interpretative complexity have to be encouraged. Furthermore, the present study followed the practice of offering data analysis programs for singlecase designs in freeware, such as R (see, e.g., Bulté \& Onghena, 2008) - a practice that we feel ought to be promoted.

The present investigation focused only on $\mathrm{AB}$ designs, although the results are potentially applicable to multiplebaseline designs (Busse, Kratochwill, \& Elliott, 1995). The data sets used in the present study were constructed using permanent linear trend, constant variance, and constant autocorrelation throughout the whole series. These data assumptions are common to simulation studies on $N=1$ designs (see, e.g., Brossart et al., 2006; Huitema \& McKean, 2007a, 2007b; Matyas \& Greenwood, 1990; Parker \& Brossart, 2003). Thus, future studies may explore the performance of PNCD for ABAB designs with curvilinear trends, computing the percentage for each change in the condition, as was suggested by Kromrey and Foster-Johnson (1996). Additionally, comparative studies such as the present one, which center on finding the technique that performs better, need to be complemented by precision studies in order to identify techniques that perform well - that is, yield accurate estimates of the effect sizes simulated.

\section{AUTHOR NOTE}

The present research was supported by the Comissionat per a Universitats i Recerca del Departament d'Innovació, Universitats i Empresa of the Generalitat de Catalunya, and the European Social Fund. The authors thank the anonymous reviewers for their useful comments and suggestions, which contributed to improving the manuscript. Correspondence concerning this article should be addressed to R. Manolov, Departament de Metodologia de les Ciències del Comportament, Facultat de Psicologia, Universitat de Barcelona, Passeig de la Vall d'Hebron, 171, 08035-Barcelona, Spain (e-mail: rrumenov13@ub.edu).

\section{REFERENCES}

Allison, D. B., \& Gorman, B. S. (1994). "Make things as simple as possible, but no simpler": A rejoinder to Scruggs and Mastropieri. Behaviour Research \& Therapy, 32, 885-890.

Beretvas, S. N., \& Chung, H. (2008). An evaluation of modified $R^{2}$-change effect size indices for single-subject experimental designs. Evidence-Based Communication Assessment \& Intervention, 2, 120-128.

BRADLEY, J. V. (1977). A common situation conducive to bizarre distribution shapes. American Statistician, 31, 147-150.

Brossart, D. F., Parker, R. I., Olson, E. A., \& Mahadevan, L. (2006). The relationship between visual analysis and five statistical analyses in a simple $\mathrm{AB}$ single-case research design. Behavior Modification, 30, 531-563.

Bulté, I., \& Onghena, P. (2008). An R package for single-case randomization tests. Behavior Research Methods, 40, 467-478.

Busse, R. T., Kratochwill, T. R., \& Elliott, S. N. (1995). Metaanalysis for single-case consultation outcomes: Applications to research and practice. Journal of School Psychology, 33, 269-285.

CoHEn, J. (1990). Things I have learned (so far). American Psychologist, 45, 1304-1312.

CoHen, J. (1994). The earth is round $(p<.05)$. American Psychologist, 49, 997-1003.

CRANE, D. R. (1985). Single-case experimental designs in family therapy research: Limitations and considerations. Family Process, 24, 69-77.

GEDo, P. M. (2000). Single case studies in psychotherapy research. Psychoanalytic Psychology, 16, 274-280.

Greenwood, K. M., \& Matyas, T. A. (1990). Problems with application of interrupted time series analysis for brief single-subject data. Behavioral Assessment, 12, 355-370.

Harrop, J. W., \& Velicer, W. F. (1985). A comparison of alternative approaches to the analysis of interrupted time-series. Multivariate Behavioral Research, 20, 27-44.

Huitema, B. E., \& McKean, J. W. (2000). Design specification issues in time-series intervention models. Educational \& Psychological Measurement, 60, 38-58.

Huitema, B. E., \& McKean, J. W. (2007a). Identifying autocorrelation generated by various error processes in interrupted time-series progression designs: A comparison of AR1 and portmanteau tests. Educational \& Psychological Measurement, 67, 447-459.

Huitema, B. E., \& McKean, J. W. (2007b). An improved portmanteau test for autocorrelated errors in interrupted time-series regression models. Behavior Research Methods, 39, 343-349.

Huitema, B. E., McKean, J. W., \& McKnight, S. (1999). Autocorrelation effects on least-squares intervention analysis of short time series. Educational \& Psychological Measurement, 59, 767-786.

Jenson, W. R., Clark, E., Kircher, J. C., \& Kristjansson, S. D. (2007). Statistical reform: Evidence-based practice, meta-analyses, and single subject designs. Psychology in the Schools, 44, 483-493.

KAZDIN, A. (1978). Methodological and interpretive problems of singlecase experimental designs. Journal of Consulting \& Clinical Psychology, 46, 629-642.

KIRK, R. E. (1996). Practical significance: A concept whose time has come. Educational \& Psychological Measurement, 56, 746-759.

Kratochwill, T. R., \& Brody, G. H. (1978). Single subject designs: A perspective on the controversy over employing statistical inference and implications for research and training in behavior modification. Behavior Modification, 2, 291-307.

Kromrey, J. D., \& Foster-Johnson, L. (1996). Determining the efficacy of intervention: The use of effect sizes for data analysis in singlesubject research. Journal of Experimental Education, 65, 73-93.

MA, H. H. (2006). An alternative method for quantitative synthesis of single-subject research: Percentage of data points exceeding the median. Behavior Modification, 30, 598-617.

Manolov, R., \& Solanas, A. (2008). Comparing $N=1$ effect size indices in presence of autocorrelation. Behavior Modification, 32, 860-875.

Manolov, R., Solanas, A., \& Leiva, D. (in press). Comparing "visual" effect size indices for single-case designs. Methodology-European Journal of Research Methods for the Behavioral \& Social Sciences.

Matyas, T. A., \& GreEnwoOd, K. M. (1990). Visual analysis for singlecase time series: Effects of variability, serial dependence, and mag- 
nitude of intervention effects. Journal of Applied Behavior Analysis, 23, 341-351.

McCleary, R., \& Hay, R. A., JR. (1980). Applied time series analysis for the social sciences. Beverly Hills, CA: Sage.

McGrath, R. E., \& Meyer, G. J. (2006). When effect sizes disagree: The case of $r$ and $d$. Psychological Methods, 11, 386-401.

MicCERI, T. (1989). The unicorn, the normal curve, and other improbable creatures. Psychological Bulletin, 105, 156-166.

Parker, R. I., \& Brossart, D. F. (2003). Evaluating single-case research data: A comparison of seven statistical methods. Behavior Therapy, 34, 189-211.

Parker, R. I., Brossart, D. F., Vannest, K. J., Long, J. R., Garcia De-Alba, R., Baugh, F. G., \& Sullivan, J. R. (2005). Effect sizes in single case research: How large is large? School Psychology Review, 34, 116-132.

Parker, R. I., Cryer, J., \& Byrns, G. (2006). Controlling baseline trend in single-case research. School Psychology Quarterly, 21, 418-443.

Parker, R. I., Hagan-Burke, S., \& Vannest, K. (2007). Percentage of all non-overlapping data: An alternative to PND. Journal of Special Education, 40, 194-204.

Rosnow, R. L., \& Rosenthal, R. (1989). Statistical procedures and the justification of knowledge in psychological science. American Psychologist, 44, 1276-1284.

Schlosser, R. W., Lee, D. L., \& WendT, O. (2008). Application of the percentage of non-overlapping data (PND) in systematic reviews and metaanalyses: A systematic review of reporting characteristics. EvidenceBased Communication Assessment \& Intervention, 2, 163-187.
Schlosser, R. W., \& Sigafoos, J. (2008). Meta-analysis of singlesubject designs: Why now? Evidence-Based Communication Assessment \& Intervention, 2, 117-119.

Schneider, N., Goldstein, H., \& Parker, R. (2008). Social skills interventions for children with autism: A meta-analytic application of percentage of all non-overlapping data (PAND). Evidence-Based Communication Assessment \& Intervention, 2, 152-162.

Scruggs, T. E., \& Mastropieri, M. A. (1998). Summarizing singlesubject research: Issues and applications. Behavior Modification, 22, 221-242.

Scruggs, T. E., Mastropieri, M. A., \& Casto, G. (1987). The quantitative synthesis of single-subject research: Methodology and validation. Remedial \& Special Education, 8, 24-33.

Shadish, W. R., Rindskopf, D. M., \& Hedges, L. V. (2008). The state of the science in the meta-analysis of single-case experimental designs. Evidence-Based Communication Assessment \& Intervention, 2, 188-196.

SYMON, J. B. (2005). Expanding interventions for children with autism: Parents as trainers. Journal of Positive Behavior Interventions, 7, 159-173.

Tervo, R. C., Estrem, T. L., Bryson-Brockman, W., \& Symons, F. J. (2003). Single-case experimental designs: Application in developmental-behavioral pediatrics. Developmental \& Behavioral Pediatrics, 24, 438-448.

Wilkinson, L., \& THE TASk Force On Statistical Inference (1999). Statistical methods in psychology journals: Guidelines and explanations. American Psychologist, 54, 694-704.

\section{APPENDIXA}

An example of R code computing PND and PNCD as output. The input required from the user is (1) the data for phase $A$ in the expression phaseA $<-c(1: 10)$, replacing " $1: 10$ " with the measurements obtained separated by commas; and (2) the data for phase B placed instead of " $11: 20$ " in the expression phaseB $<-C(11: 20)$. After introducing the behavioral measurements, the text is copied and pasted into the $\mathrm{R}$ console, and the estimates are printed out.

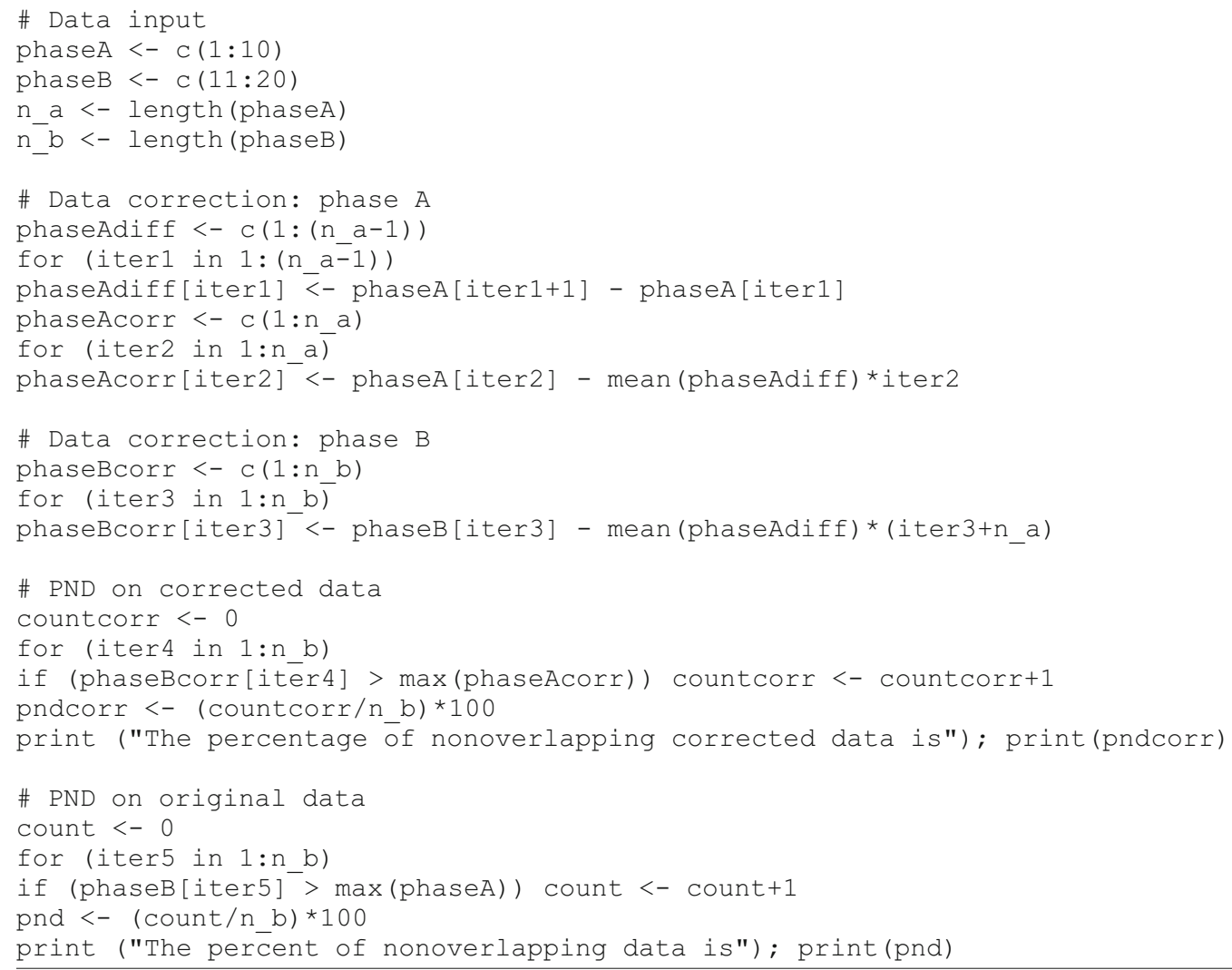




\section{APPENDIX B}

R code computing PND and PNCD as output used, as is described in Appendix A. This is useful when the objective of the behavior of interest is an undesirable one and the treatment pretends to eliminate or reduce it.

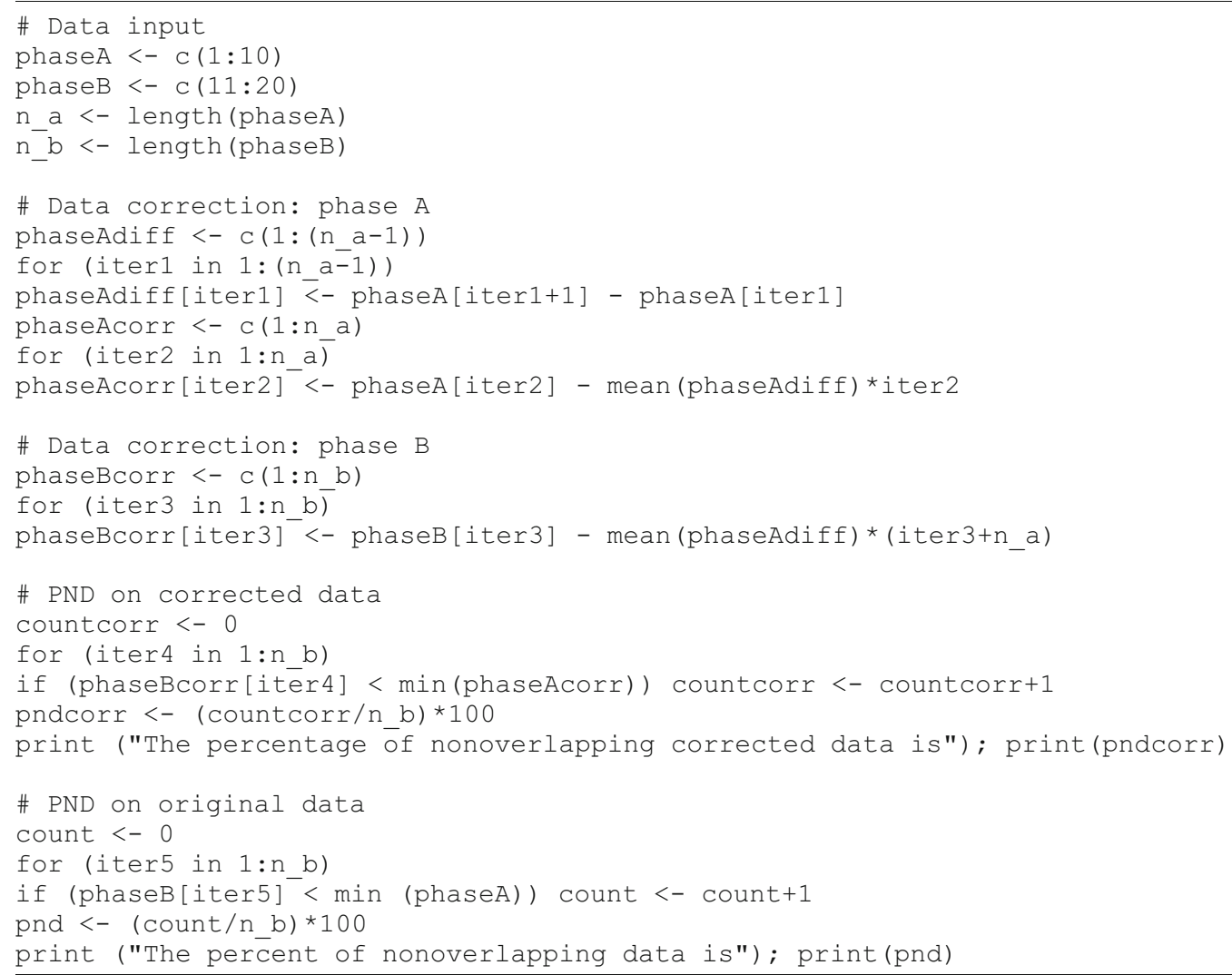

(Manuscript received February 26, 2009;

revision accepted for publication July 14, 2009.) 